\title{
Features of the Value Function for Voice and Their Consistency across Participants from Four Countries: Great Britain, Mexico, The Netherlands, and the United States
}

\author{
Kenneth H. Price \\ Department of Management, University of Texas at Arlington \\ Thomas W. Hall \\ Department of Accounting, University of Texas at Arlington
}

Kees van den Bos

Department of Social Psychology, Free University Amsterdam, Amsterdam, The Netherlands

J ames E. Hunton

School of Accountancy, University of South Florida

Steve Lovett

Department of Management, San Diego State University at Imperial Valley

and

Mark J . Tippett

School of Business \& Economics, University of Exeter, Exeter, England, United Kingdom

This study investigated features of the value function for voice using subjects from four countries: Great Britain, Mexico, The Netherlands, and the United States. Across these four groups of subjects the shape of the value function was found to be similar, though differences in the estimated reference points were

The research reported in this article was supported in part by a fellowship of the Royal Netherlands Academy of Arts and Sciences awarded to Kees van den Bos.

Address correspondence and reprint requests to Dr. Kenneth H. Price, Department of Management, UTA Box 19467, Arlington, Texas, 76013. E-mail: price@uta.edu. 


\begin{abstract}
detected. Consistent with predictions derived from prospect theory (Kahneman \& Tversky, 1979) the relationship between the value of voice and the magnitude of voice was found to be direct, monotonic, and nonlinear. The largest increment in value occurred when the magnitude of voice shifted from mute to some voice. Thereafter, increments in valuetended to decline in magnitude suggesting diminishing marginal returns on the response measure of procedural fairness. An unexpected finding was that the final segment of the value function was convex indicating increasing marginal returns as the magnitude of voice shifted from its penultimate level to its maximum possible level. The study also investigated whether subjects' reported expectations of voice correspond to the value function reference point as theorized in the literature. Findings suggest that self-reported expectations of voice are higher than the estimated value function reference point.

2000 Academic Press
\end{abstract}

The positive effects of voice (i.e., when a decision maker considers the concerns, preferences, and interests of participants influenced by the decision) are well documented in the literature. The exercise of voice is associated with increased perceptions of fairness and control in studies of performance appraisals (Greenberg, 1990), employee compensation (Miceli \& Lane, 1991), survivors' reactions to layoffs (Brockner \& Greenberg, 1990), managerial dispute resolution (Karambayya \& Brett, 1989), goal setting, (Earley \& Lind, 1987), and decision making (Lind, Kanfer, \& Earley, 1990). In turn, increases in fairness areassociated with decreased turnover and increased job satisfaction, organizational commitment, trust in supervision, and task performance (Brockner \& Wiesenfeld, 1996; Greenberg, 1990; Hunton \& Price, 1997; Lind et al., 1990). Increases in perceptions of fairness and control, as well as task performance, can also occur when participants exercisechoice; that is, when a decision maker allows participants to select the outcome (e.g., Folger \& Cropanzano, 1998; Greenberg \& Folger, 1983; Hunton \& Price, 1997).

Theory and research on voice and choice have also examined when and how participant input should be solicited. From a participant's perspective, both of these questions are related to the value of the voice solicitation. From a perspective of when voice should be solicited, Maier (1973) argues that if fairness in the distribution of outcomes is of central concern, participants should be involved in the decision. Vroom and J ago (1988) suggest that when autocratic decisions are not likely to be accepted, allowing participation is likely to increase commitment to the decision. Strumpf, Zand, and F reedman (1979) document that when representative decisions are needed (i.e., decisions affecting a large number of different constituents) participatory decision modes should be used. With respect to how voice should be solicited, the genuineness of the voice solicitation is of critical concern. If participants feel that a decision maker fails to give participant input adequateconsideration, or if the voice solicitation is perceived as disingenuous, the outcome is likely to be frustration and resentment (e.g., Baldwin, Magjuka, \& Loher, 1991; Folger, 1977). 
Questions regarding the value of voice from a participant's perspective are the central focus of this research. Specifically we are interested in (1) the relationship between voice magnitude and the value of the voice solicitation (the value function for voice) and (2) the consistency of this relationship across four countries: Great Britain, Mexico, The Netherlands, and the United States. We also use the experimental data to estimate the value function reference points for subjects from each country and explore the relationship between these estimates and two other measures: (1) participants' self-reported expectations of voice and (2) a measure of participants' attitudes toward decision centralization. The next section reviews studies where the magnitude of voice solicitation has been examined. In these studies voice was solicited in an appropriatecontext, thesolicitation of voicewas not disingenuous, and the magnitude of voice refers to the amount of voice that was solicited.

\section{MAGNITUDE AND VALUE OF VOICE}

A number of studies examining the impact of the magnitude of voice suggest that more voice is better. F or some researchers, magnitude refers to voice which spans different phases of the decision-making process. Voice across decision phases can also reflect changes in the qualitative as well as the quantitative amount of voice. Cooper and Wood (1974) found that voice in all stages of decision making (planning through postimplementation) resulted in more positive perceptions of involvement than voice occurring in only one stage. Earley and Lind (1987) report that participation early in the decision process may be beneficial when such voice involves the selection of alternatives. They argue that choice offered later in the decision process may not be meaningful if the options from which a participant can choose are not desirable. Hunton, Price, and Hall (1996) report that the combination of predecisional and postdecisional voice, compared to predecisional voice, enhances the perceived fairness of the decision process.

Additional research suggests, however, there are conditions that can place limits on when more voice is better. These studies document a number of complexities regarding the conditions or combined set of conditions when voicebased participation is likely to result in a fair process effect. Research by Folger (1977) reports that decision makers who allow participants voice, compared to a mute condition, are perceived as less fair when inequitable outcomes are changed to be more equitable. However, when the inequitable allocation is maintained, decision makers who allow voice, compared to a mute condition, are perceived as more fair. Other research has reported that positive effects of voice can depend on how voice-based participation is solicited (e.g., Folger \& Cropanzano, 1998). If the solicitation of voice is perceived to be disingenuous, for example the decision maker does not give a participant's concerns and preferences due consideration, then the exercise of voice can result in frustration. Research by Van den Bos, Vermunt, and Wilke (1996) reports that participants who expect no voice, yet receive voice, perceive the process as less fair and perform at lower levels than participants who neither expect nor receive 
voice. Examining the impact of higher levels of voice, when expectations for voice were not violated, Hunton, Hall, and Price (1998) found that increases in the magnitude of voice resulted in diminishing marginal returns. In samples of experienced financial managers and business students, higher levels of voice were associated with positive but declining increases on response measures of fairness, control, and satisfaction. Recent research by Peterson (1999) continued this theme examining voice in small decision-making groups. He reported that stricter decision rules, while allowing higher levels of voice, resulted in lower levels of group member satisfaction. This occurred because stricter decision rules were accompanied by higher levels of substantive conflict.

In the present study, we continue the investigation of the complexities of voice-based participation by examining the valuefunction for voiceusing propositions from prospect theory. More specifically, we argue that the work by Kahneman and Tversky (1979) on prospect theory can hel p explain and predict the impact of changes in the magnitude of voice on the value of the voice solicitation. Other justice theorists have also applied constructs of Kahneman and Tversky to devel op theory and guide research in procedural justice. Folger (1986, 1987) incorporates the use of Kahneman and Tversky's (1982) simulation heuristic to explain reactions to outcomes and procedures. Consequently, this research follows a tradition that has been established in the procedural justice literature. Based on these considerations, Kahneman and Tversky's (1979) work on prospect theory is reviewed in the next section. In adopting this framework, we do not mean to imply that voice does not have symbolic meaning (e.g., Lind, Kanfer, \& Earley, 1990). Theory and research consistent with the relational model of procedural justice have documented that the solicitation of voice increases perceptions of fairness because participants feel they are being treated with politeness, dignity, and respect appropriate for full group members (e.g., Lind, 1995; Tyler \& Lind, 1992). Rather, in using a prospect theory framework, we are simply examining the multifaceted nature of voice-based participation.

Prospect Theory: Voice as a Commodity and the Potential Role of Expectations

Prospect theory (Kahneman \& Tversky, 1979) suggests that individuals assess the value of alternative amounts of a commodity (e.g., money and time off from work) in relation to a neutral reference point. The rationale for this assertion is that human perception is attuned to the detection of change in a stimulus rather than to the absolute magnitude of the stimulus. When an individual responds to a stimulus, the response is a function of the level of the stimulus and the individual's reference point. In essence, the current stimulus is compared to the reference point and coded as falling above or below the reference point. Stimulus outcomes that compare favorably to the reference point are perceived as favorable outcomes while stimulus outcomes that compare unfavorably to the reference point are perceived as unfavorable outcomes. 
Hence, thereference point is the amount of commodity received that theindividual would consider as a neutral outcome that is neither a gain nor a loss.

In discussing the reference point, Kahneman and Tversky (1979) suggest that it may be the level of a particular stimulus to which the individual has adapted. Usually, this is the level of the stimulus that the individual has experienced in the past and present and is considered the status quo. In some circumstances, however, thereference point may bean expectation or aspiration level (Kahneman \& Tversky 1979, p. 286). This can occur where the individual has adapted to an expectation or aspiration level that is different from the current status. For example, an individual who expects to receive a raise of $\$ 5,000$ may use this as a reference point. In this circumstance, a raise of $\$ 3,000$ may be perceived as a loss because it falls below the reference point (Kahneman, 1992).

Another important feature of the prospect theory value function is that as the amount of the commodity received moves further away from the reference point, the marginal significance or value associated with unit increments in the amount of the commodity received declines. Thus, larger and larger quantities of the commodity are required to produce uniform changes in perceived value. Kahneman and Tversky (1979) use a general psychological principle, based on Weber's (1834) law, to account for this property of diminishing marginal returns. Weber's (1834) law states that larger amounts of a stimulus change are needed to detect change as the magnitude of the stimulus increases. As an example, they suggest that it is easier to discriminate between a change of $3^{\circ}$ and $6^{\circ}$ than between a change of $13^{\circ}$ and $16^{\circ}$ (Kahneman \& Tversky, 1979, p. 278). Kahneman and Tversky propose that the same principle is consistent with the evaluation of other commodities, such as money, and point to supportive research by Galanter (1962). The presence of diminishing marginal returns means that the value function is concave above the reference point and convex below the reference point. Consequently, the value function takes on an $\mathrm{S}$ shape due to the principle of diminished marginal significance. A hypothetical value function is shown in Fig. 1. As shown in this figure, the commodity value function is nonlinear and monotonic, with declining changes in value as the

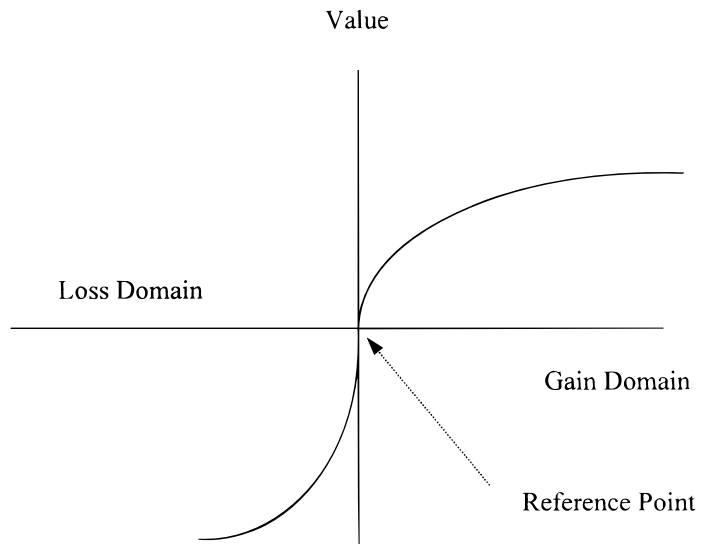

FIG. 1. Hypothetical value function for a commodity. 
amount of the commodity received moves further and further from the reference point.

\section{Alternatives to Prospect Theory}

The process of comparing current experiences to a standard is not unique to prospect theory. Other researchers have used this comparison process to explain perceptions of job satisfaction (e.g., Locke, 1969), perceptions of the quality of life (Rice, McF arlin, Hunt, \& Near, 1985), and the occurrence of job strain (French, Caplan, \& Harrison, 1982). Locke (1969) suggests that the experience of job dissatisfaction and job satisfaction are a function of the relationship between what one wants from a job and what one perceives the job as offering. What one wants from a job is a value standard against which comparisons are made of current offerings. Theorizing by Rice, McF arlin, Hunt, and Near (1985) posit that the quality of life for a specific domain (e.g., family and work) is a function of outcomes in each domain compared to a standard (e.g., aspirations, expectations, values, and needs). The perceived quality of life for each domain combines additively to determine the overall quality of life. Similarly, French et al. (1982) use a theory of person-environment fit to explain job strain. Person-environment fit involves the degree of discrepancy between the motives of the person and the supplies offered by the job to satisfy these motives.

Common to prospect theory and the work of Locke (1969), Rice et al. (1985), and French et al. (1982) is the nature of the discrepancy that results from comparing current experiences against the standard. Outcomes that fall below thestandard (e.g., based on what one wants and what oneactually experiences) are states of deficiency, while outcomes falling above the standard are states of excess. Where prospect theory and other theorists diverge is in explaining the functional form of the relationship between the experience-standard discrepancy and outcomes such as job satisfaction and job strain. Prospect theory predicts an S-shaped curve with diminishing (but positive) marginal returns as the outcome moves further from the reference point as the state of deficiency or excess increases. In explaining job satisfaction and job strain, theorists (e.g., Rice et al., 1985; French et al., 1982) indicate the form of the function can be similar to that proposed by prospect theory. However, in contrast to prospect theory they suggest that the function might also be linear (constant marginal returns) or an inverted $U$ (declining positive marginal returns up to a point and thereafter increasing negative returns). Locke (1969) suggests the form of function may vary based on whether, for example, excess deviations from the standard are disliked (negative marginal returns) or valued (positive marginal returns). For Rice et al. (1985) the form of the function may vary based on the domain being considered, the outcome being appraised, the type of standard being applied, the characteristics of the individual, or some combination of these factors. For French (1982) critical factors include whether supplies for one motive can be used as supplies for or reduce the supply for other motives. Thus, different theorists agree that judgments are made by reference to a 
standard, but diverge in expectations about whether the function's marginal returns will be positive, negative, or zero and whether these returns will be constant, increasing, or decreasing.

\section{Voi ce Research and Prospect Theory}

Treating voice as a commodity can help to explain the value of voice across decision stages. As applied to the previously cited research (e.g., Cooper \& Wood, 1974; Earley \& Lind, 1987), participants may perceive that voice should span different phases of the decision cycle. Issues across different phases can be of direct concern to the participants and/or the meaningfulness of later participation may be dependent on initial participation (Earley \& Lind, 1987). Consequently, under these conditions, more voice may be accompanied by incremental increases in the value of voice when initial solicitations of voice fall within the domain of losses. When the quantitative amount of voice exceeds expectations, more voicemay be better, al though accompanied with diminishing marginal returns (Hunton et al., 1998). Qualitatively, there is less to be gained from each increment of voice. Consequently, expectations regarding the amount of voice that should be solicited form boundaries for judgments regarding the value of the voice solicitation.

Research cited earlier, by Hunton et al. (1998), directly supports theorizing by Kahneman and Tversky (1979). Hunton et al. (1998) reported that increases in the magnitude of voice were associated with declining increases in the value of voice. However, the research by Hunton et al. (1998) did not assess participants' expectations. Consequently, the role played by expectations in the value function for voice is unknown. Other justice research, while not designed to assess the possibility of diminishing marginal returns of voice, has indicated the importance of expectations in evaluating the fairness of a procedure. A study by Doll and Torkzadeh (1988) report that participation efforts were dysfunctional when desired participation exceeded actual participation. Folger, Rosenfield, and Robinson (1983) created the expectation among participants that a specific procedure would be used. When violations of the expected procedure occurred, in the absence of adequate justification, resentment resulted. Finally, Coalter and Price (1997) examined the impact of expectations from the perspective of organizational traditions. Using a scenario approach, they reported that mute, voice, and choice procedures were perceived as more fair when consistent with organizational traditions. Consequently, the value associated with different levels of voice may depend on the participants' expectations. Voice that exceeds expectations may be perceived as a gain and voice that falls below expectations may be seen as a loss.

Thecurrent study seeks to replicateand extend previous research examining the value function for voice (Hunton et al., 1998). Consistent with this goal, we expect to replicate results of earlier studies that document a nonlinear value function for voice. Our first hypothesis is as follows: 
Our research also addresses the cross-cultural generality of the features of the voice value function. Examining the effects of cultural influences is important in defining boundary dimensions that influence outcomes associated with voice-based participation and in facilitating the development of a broad theoretical framework for understanding procedural justice. Additionally, cultural differences are a particularly important consideration given increasing economic and political interdependency among countries. Our expectations are that the nonlinear form will be similar across different cultures, but the value function reference point will vary to the extent that different norms of participation exist across the four countries. The reasons for our expectations and our specific hypotheses concerning cross-cultural comparisons are explained in the next two sections.

\section{Generality of the Voice Value Function across Countries}

As described earlier, Kahneman and Tversky (1979) use general psychol ogical principle (Weber's law) to account for the property of diminishing marginal returns in a value function. Coren, Ward, and Ellis (1994, pp. 42-43) also suggest that Weber's law provides a good fit with the data when examined across a number of different sensory modalities. Additionally, research on social psychological processes provides evidence for diminishing marginal returns in different contexts. In examining the effects of group size, Latané (1981) cites research where increases in group size had proportionally smaller effects (e.g., conformity, stuttering, and tipping). Consequently, evidence for diminishing marginal returns appears to be robust across different psychophysical, as well as social psychological, phenomena. To the extent that the underlying mechanism for diminishing marginal returns is a basic psychological process, there would be support for a nonlinear value function across cultures if the respondents value the exercise of voice.

Other studies provide support that the exercise of voice will be associated with fair procedures across cultures when examining preferences for dispute resolution procedures among participants in Hong Kong and the U nited States (Leung \& Lind, 1986) and in the United States, Germany, Hong Kong, and J apan (Lind, Tyler, \& Huo, 1997). These studies suggest that the processes driving procedural justice judgments are similar across cultures. Results of the former study document that participants in both the United States and Hong Kong who preferred an adversarial style to settling disputes did so because of the process control it offered. However, participants in Hong Kong, when compared to the United States, did not perceive the adversarial technique as more desirable than the nonadversarial technique. The researchers argued that the downside of adversarial techniques, thelikelihood that they exacerbate the conflict, negated the positive beneficial use of increased process control.

The latter study by Lind et al. (1997) examined antecedents of fairness in dyadic and third-party conflict resolution procedures across three cultures. Testing examined whether the relational model of procedural justice(i.e., status recognition, neutrality, and trust in benevolence; see Tyler \& Lind 1992, for a 
more complete introduction to the relational model) could account for perceptions of fairness. Results indicated that the relational model could account for voiced-based effects on perceptions of fairness across the four cultures. In addition, there was considerable similarity across cultures in interpretation of which factor (i.e., status recognition, neutrality, and trust in benevolence) contributed to the perceived fairness of a specific dyadic versus third-party conflict resolution technique.

In summary, Weber's law and studies reporting that factors driving fairness perceptions are similar across cultures suggest a nonlinear value function for voice across cultures. Based on the available literature indicating the applicability of prospect theory to explain the value of voice-based participation and the similarity in the interpretation of voice across cultures our second hypothesis is as follows:

$\mathrm{H}$ 2: The voice value function is expected to assume the same form across all cultures.

\section{Expected Differences in Value Function Reference Points}

As noted earlier, Kahneman and Tversky (1979) theorize that the reference point used in assessing the value of an outcome may bebased on an individual's past or present experiences, expectations, or aspirations. Consequently, it seems reasonable to expect that the voice value functions estimated in the current study will exhibit reference points that vary according to cultural norms of participation. Research by Hofstede (1980) has identified four dimensions that differentiate cultures. The basic factor structure has been confirmed by Hoppe (1998) using a different sample and in reviews of the literature (Sondergaard, 1994). One of these dimensions, power distance, is a characteristic of a culture and defines the extent that the less powerful persons in a society accept inequality in power and consider it normal (H ofstede, 1984). Using the concept of power distance it is possibleto measure the degree of "authoritarianism" of the dominant supervisory styles (Hofstede, 1980). Countries that are high in power distance are more hierarchical and their employees are less likely to disagree with their supervisors and more likely to perceivethat decisions are, and perhaps should be, made in an autocratic way. Consequently, individuals from countries that rate high on the power distance index are likely to be more accepting of lower levels of participation in workplace decisions and thus have lower expectations of participation.

Studies examining cultural differences that pertain to participation and the exercise of voice have been reviewed by McF arlin and Sweeney (1998). Supporting Hofstede's research on country differences in power distance, McF arlin and Sweeney (1998) conclude that voice-based procedures in the United States increase the perceptions of the fairness of the appraisal process. However, in reviewing the work of McEvoy and Cascio (1990), they note that autocratic appraisal processes are more acceptable in Taiwan and that Tai wanese employees are less likely to provide input regarding their appraisals. They also cite 
the work of McF arlin, Sweeney, and Cotton (1993) in support of cultural differences in participation. They note that due to culture differences, participative management strategies have not been successful in some European countries. Consistent with thesefindings, research by Stohl (1993) reported that interpretation of the meaning of participation by managers was consistent with country differences in power distance as well as uncertainty-avoidance, which is another factor of cultural differentiation identified by Hofstede (1980). For example, participation was viewed by managers from France, a higher power distance country, as a centralized process emanating through hierarchical levels, whilemangers from Denmark, a low power distance country, view participation as a more spontaneous activity evolving from the daily interactions of superiors and subordinates. Looking at empowerment, a strategy emphasizing the decentralization of power, Eylon and Au (1999) report that individuals from high and low power distance countries did not differ in job satisfaction when empowered. However, participants from high power distance countries did not perform as well when empowered compared to being disempowered; participants from low power distance countries did not differ in performance as a function of empowerment.

Other research studies, while not explicitly dealing with voice or participation, provide indirect support for country differences in power distance influencing voice or the extent of participation. Earley (1999) reports that judgments of team efficacy and performance were more strongly tied to perceptions of high status members in high power distance countries, while perceptions of team efficacy did not differ by member status in low power distance countries. Earley speculates that in high power difference countries, the high-status member may have anchored the performance goal and low-status members fulfill the high-status member's expectations. Country differences in power distance wereal so related to preferences to gain support from those in authority prior to taking action on an innovation (Shane, Venkataraman, \& MacMilan, 1995) and lower levels of reliance on coworkers to handle disagreements (Smith, Dugan, Peterson, \& Leung, 1998). Consequently, the above set of findings suggests that one aspect of cultural differentiation, power distance, is consistently tied to variations in and reactions to participation.

In the current study, the value function of voice is examined using samples of participants from Great Britain, Mexico, The Netherlands, and the United States. Hofstede (1980, p. 74) reported that the largest power difference between countries examined in this study is between M exico versus Great Britain, The N etherlands, and the U nited States, with the latter three countries closely grouped together. Kras (1995) also examined supervisory-subordinate relations in Mexico and the United States. His findings converge with Hofstede supporting lower expectations for voice among participants in Mexico. Given cultural differences in expectations for participation our third hypothesis is as follows: 
from Mexico compared to participants from Great Britain, The Netherlands, and the United States. ${ }^{1}$

\section{Participant Expectations and the Voice Value Function Reference Point}

In addition to the prior hypotheses, given the theoretically important role of a reference point in understanding the voice value function, we investigate whether participants' expectations, unmeasured in previous research, correspond to the estimated value function reference point. Because there are many possible expectation constructs, as described in Measures of Participants' Expectations under Method, this research issueis exploratory in nature. Ther efore no specific hypothesis is tested.

\section{METHOD}

Participants, Procedures, and Materials

Participants were students enrolled at four universities. In Great Britain ( $N=118$ ), the modal age was 21 or less, $67 \%$ worked at least part-time, $56 \%$ were 2 nd-year college students, and $56 \%$ were male. In Mexico ( $N=185)$, the modal age was 21 or less, $47 \%$ worked at least part-time, $33 \%$ were seniors, and $56 \%$ were female. In The Netherlands $(\mathrm{N}=99)$, the modal age was also 21 or less, $49 \%$ worked at least part-time, 58\% were 1st-year students, and $71 \%$ were female. In the United States $(\mathrm{N}=109)$, the modal age was again 21 or less, $87 \%$ worked at least part-time, $41 \%$ were 2 nd-year students, and $50 \%$ were male.

In each university, the experiment was conducted over multiple sessions. Participants were randomly assigned to experimental conditions and received one of six versions of a case scenario used by Hunton et al. (1998). In The Netherlands, the scenario materials wereadministered using computers, while in the remaining countries the materials were administered in written form. The materials were originally written in English and then translated into Spanish and Dutch by researchers who were native speakers of the languages and members of the research team. Materials were translated back to English to provide an additional check on the fidelity of the translation. The materials could be completed in approximately $30 \mathrm{~min}$.

Participants were asked to assume the role of account managers. Due to rapid technol ogical advances, their companies were updating their companies' computer systems, peripheral devices, and software. Participants were told they had seen a list of 20 different features of the new systems (e.g., type of processor and type of printer) and told to assume that each of the potential

\footnotetext{
${ }^{1}$ In the sample of countries we examined, rankings based on two other dimensions identified by Hofstede (1980), individualism and uncertainty avoidance, could also be used to predict the same ordering of reference points as provided by the power distance measure. We chose to rely on the power distance measure since this dimension relates most directly to the concept of voicebased participation.
} 
features on the list were equally important. Furthermore, budget constraints could force trade-offs in the selection of computer system attributes. A fast hard drive could leave less money to spend for software. Their supervisor would make the final purchase decision from this list. Additional case information informed the participants that they spent $85 \%$ of their work day using their personal computer, and since their current system was outdated, acquisition of the new computers were an eagerly anticipated event.

\section{Manipulation of Independent Variables}

Five versions of the case materials manipulated the magnitude of participant voice (magnitude of voice condition). Participants weretold either their supervisor had not asked them to express their opinions and preferences for any of the 20 choices (mute condition) or they had been asked for their opinions and preferences for $5,10,15$, or 20 of the 20 features of the new computer system. Participants in these five conditions were asked for their reactions to the manipulated level of voice. The sixth version of the case was designed to measure participant expectations of voice (expectation condition). Case materials were the same for these participants with the following exception; they were told that their supervisor could request their opinions and preferences for the computer choices that needed to be made. These participants answered questions measuring their expectations of what their supervisor might do in soliciting input.

Dependent Measures for Magnitude of Voice and Manipulation Check Questions

All dependent measures were assessed using 9-point bipolar scales, with a minimum value of 1 and a maximum value of 9 . I tems measuring reactions to no voice (mute condition) or when input was solicited to 5, 10, 15, or 20 of 20 alternatives were adopted from other justice studies (e.g., E arley \& Lind, 1987; Lind et al., 1990). Threequestions measured perceptions of procedural fairness. Participants indicated how unfair to fair and unjust to just the procedures were in determining the new computer and the extent they were satisfied with the procedures used to determine the computer system (strongly disagree to strongly agree). These items were combined into a single scale. For outcome control, questions asked the extent to which the respondents' preferences determined (no extent to great extent), influenced (no influenceto completeinfluence), and controlled (no control to complete control) the configuration of the new computer system. Three questions measuring anticipated outcome satisfaction were adopted from Doll and Torkzadeh (1988). Participants were asked to anticipate their degree of satisfaction (completely dissatisfied to completely satisfied) with their new computer system, their feelings toward (not satisfied to completely satisfied) their new computer system, and whether they will be satisfied with their new computer system (strongly disagreeto strongly agree). Estimates of reliability, using coefficient alpha $(N=511)$, for the scales of 
fairness, control, and satisfaction were $\alpha=.88, \alpha=.87$, and $\alpha=.93$, respectively. Corresponding reliability indices for each country were similar. They ranged across countries from .81 to .93 for fairness, from .84 to .90 for control, and .89 to .96 for outcome satisfaction.

Two manipulation check questions measured participants' ability to recall the case information. Participants were asked the amount of voice requested by their immediate supervisor and how much time they spent using their computer in a typical workday. For the first question, measuring the magnitude of voice, $95 \%$ of the respondents in The Netherlands, $92 \%$ of the respondents in the U nited States, $81 \%$ of the respondents in Mexico, and $79 \%$ of the respondents in Great Britain answered the question correctly.

For the question asking the amount of time spent working on the computer in a typical work day, all of the respondents in The Netherlands, $94 \%$ of the respondents in the U nited States, $86 \%$ of the respondents in Great Britain, and $81 \%$ of the respondents in Mexico correctly answered the question. Research findings did not significantly differ when participants incorrectly answering the questions were excluded from the analysis.

\section{Measures of Participants' Expectations in the Control Condition}

Theory developed by Kahneman and Tversky (1979) specifies that values above the reference point are coded as gains and values below the reference point as losses. Hence, the reference point plays a key role in determining the value assigned to a particular outcome. While the theory does not provide a clear-cut operationalization of the conceptual reference point, Kahneman and Tversky suggest that the reference point might be based on expectations (but there is no evidence to confirm this conjecture). Additionally, as noted earlier there is conceptual overlap between the reference point in prospect theory and the potential standards used to evaluate current job outcomes in the job satisfaction literature (e.g., Greenhaus et al., 1983). Locke (1969) suggests the state of job (dis-)satisfaction is a function of a comparison between a value standard (what is desired from a job) and what the current job is offering while Rice, McF arlin, Hunt, and Near (1985) suggest aspirations, expectations, values, and needs might be used as comparativestandards in quality-of-life perceptions.

Given the various possibilities set forth in the literature, in an attempt to identify the construct that was most consistent with the reference point participants in the control condition were asked two sets of three questions in a counterbalanced sequence. One set asked the minimum and maximum amount of input that would be acceptable as well as what participants would settle for with regard to expressing their preferences. The second set asked participants the minimum and maximum amount of input they expected their supervisor to request as well as what would probably happen. For each of the six questions, there were five possible answers. They included my supervisor would request my input for none, 5, 10, 15, or 20 of the possi ble computer system choices. Alternatives were scored from 1 to 5 , respectively. 
An additional scale measuring subjects' perceptions of decision centralization was included in this condition (Hierarchy of Authority, Aiken \& Hodge, 1966). This scale assessed where the locus of authority to make decisions was located (Cook, Hepworth, Wall, \& Warr, 1981). Participants responded to five items, from strongly disagre (1) to strongly agre (9). Sample items included supervisors have to approve decisi ons and you have to ask your supervisor before doing anything. The estimate of reliability was $\alpha=.83(\mathrm{~N}=121)$. The estimates of reliability for each country were similar in magnitude, ranging from .75 to .87 . Our purposes for collecting these data were twofold. First, following Lind et al . (1997) the inclusion of this scale provided a means to validate that participants differed in a culturally prescribed manner on a dimension of power distance as described in Hofstede (1980). Second, inclusion of the scale was expected to have explanatory value, not in explaining changes by country in the form of the voice value function, since none are expected, but to provide support for expected differences in the reference point due to variation in cultural norms of participation. We expected the highest level of decision centralization for subjects from Mexico with lower levels for subjects from Great Britain, the United States, and The Netherlands.

\section{RESULTS}

\section{Correlations among Measures}

Preliminary analysis examined the relationships among measures of procedural fairness, control, and outcome satisfaction. Correlations were .81 for the measures of fairness and control, .82 for fairness and satisfaction, and .78 for the measures of influence and satisfaction. Theory and research support an association between increases in perceptions of decision control, perceptions of procedural fairness, and outcome satisfaction (e.g., Thibaut \& Walker, 1975; Hunton \& Price, 1997). However, given the high correlation among dependent measures, and because the results did not differ by response measure, only the results for the fairness measure are reported here. ${ }^{2}$

\section{Tests of Hypotheses 1 and 2}

Based on the predictions of prospect theory, Hypothesis 1 anticipated that the value function for voice would be monotonically increasing and nonlinear across the five levels of voice (mute, 5, 10, 15, or 20 choices) examined in this study, while Hypothesis 2 predicted the same form across cultures. Initial

\footnotetext{
2 Becausethefairness scal eincluded a question that asked about satisfaction with the procedures, a second fairness scale was constructed that omitted the satisfaction question. For this second scale the Cronbach alpha was somewhat lower at .81 and the results of statistical analyses based on the second scale were materially the same as the analyses based on the first scale. Given the higher al pha for thethree-item scale, results reported in thestudy are based on the three-item scale.
} 
FEATURES OF THE VALUE FUNCTION

109

ANOVA procedures ${ }^{3}$ in a 5 (magnitude of voice) $\times 4$ (country) design revealed significant main effects for voice magnitude, $F(4,491)=64.3, p<.0001$, and country, $F(3,491)=2.9, p<.03$, but an insignificant interaction effect, $F(12$, $491)=.63, p<.82$. The absence of a significant interaction suggests that the shape of the voice value function is similar for each country (confirming Hypothesis 2) while the presence of a significant country main effect suggests that the voice value function is shifted up or down depending on the country. These effects are illustrated in Fig. 2, which plots the value function for voice

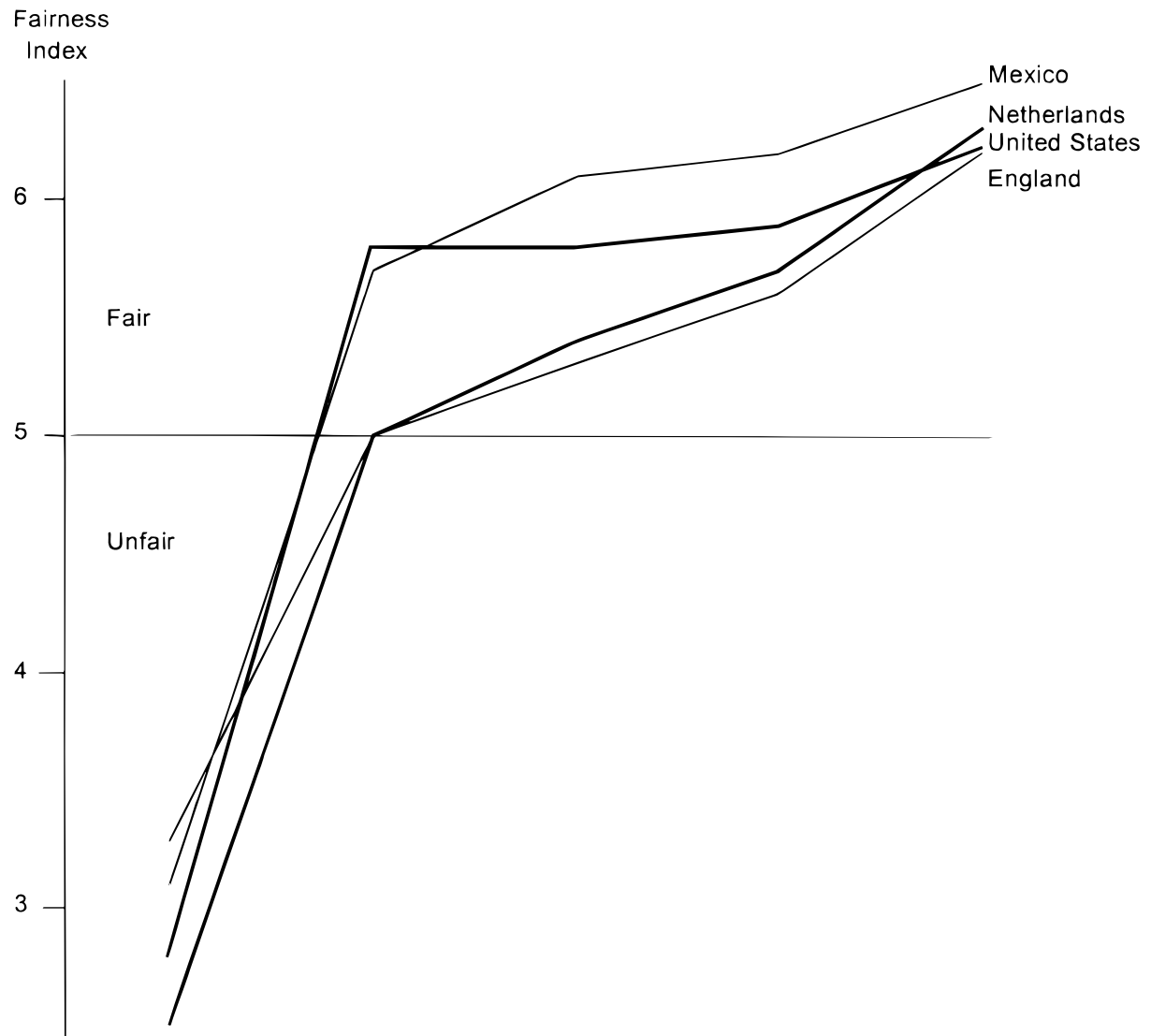

2

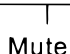

5

10

$1_{20}$

Magnitude of Voice

FIG. 2. Value functions for voice.

${ }^{3}$ The initial testing for voice magnitude effects, country effects, and interaction were conducted using ANOVA procedures rather than regression procedures because it was felt the AN OVA preentation was more parsimonious. It should be recognized, however, that a regression model with appropriate terms would produce the same results as the ANOVA. 
by country. Additionally, this figure is suggestive of a nonlinear voice value function.

To complete the testing of Hypothesis 1 , a regression analysis was used to model the relationship between the two independent variables (voice magnitude and country) and the response measure of procedural fairness. Linear, quadratic, and cubic forms of the voice magnitude variable were included in the model because these were expected given the prediction of a convex shape below the reference point and a concave shape above the reference point. Because Hypothesis 3 predicts that the voice function for subjects from Mexico will have the lowest reference point, responses for subjects from Mexico were imbedded in the intercept and indicator variables were used to identify responses of subjects from Great Britain, TheN etherlands, and theU nited States. Finally, because the prior ANOVA found no interaction, the regression model did not include any interaction terms. The full regression model was as follows:

$$
F=b_{1}+b_{2} V+b_{3} V^{2}+b_{4} V^{3}+b_{5} G+b_{6} N+b_{7} U
$$

In this model $\mathrm{F}=$ the perceived fairness response variable, $\mathrm{V}=$ the voice magnitude variable, $\mathrm{G}=$ an indicator variable identifying subjects from Great Britain, $\mathrm{N}=$ an indicator variable identifying subjects from The N etherlands, and $U=$ an indicator variable identifying subjects from the United States. Note that there is no indicator variable identifying subjects from Mexico and thus results for these respondents are reflected in the estimated intercept of the regression $\left(b_{1}\right)$.

The estimated regression function was significant, $F(6,510)=47 ; p<.0001$, and all coefficients were significant at the $\alpha=.05$ level except that the indicator variable identifying subjects from the United States was not significant. The estimated function was as follows:

$$
\mathrm{F}=3.2525+.7306 \mathrm{~V}-.06076 \mathrm{~V}^{2}+.001629 \mathrm{~V}^{3}-.4376 \mathrm{G}-.5152 \mathrm{~N}
$$

Using this estimated function we were able to test whether the voice function was concave (as expected) or convex in the region above the reference point implied by subjects' responses to the fairness measure (the next section discloses that subjects used reference points in the range of 3 to 5 on the voice magnitude scale). ${ }^{4}$ To do this we determined the second derivative of the function, $\mathrm{F}^{\prime \prime}=-.12152+.009774 \mathrm{~V}$. Then, the derivative function was evaluated for each level of voice magnitude that fell in the region above the reference

\footnotetext{
${ }^{4}$ The data that were collected did not provide a basis for examining the shape of the voice value function in region below the reference point. This limitation occurred because subjects' responses were consistent with reference points in the range of three to five choices on the voice magnitude scale of 0 to 20 . Since manipulated levels of the voice magnitude variable were $0,5,10,15$, and 20 this yielded only one observed point $(V=0)$ in the region below the reference point. To avoid this limitation in future research it may be advisable to construct cases where the reference point for voice is at a higher magnitude thus permitting a more complete examination of the voice value function.
} 
point implied by subjects' responses. By examining the sign of $F^{\prime \prime}$ we were able to determine if the voice function was convex $\left(F^{\prime \prime}>0\right)$ or concave $\left(F^{\prime \prime}<0\right)$ at a particular level of voice magnitude. At voice on 5, 10, 15, and 20 attributes, the second derivative took the following values: $F^{\prime \prime}=-.07,-.02,+.02$, and +.07 , respectively. Hence, the estimated voice value function exhibited the expected concave form at voice on 5 and 10 attributes, but changed to convex at voice on 15 and 20 attributes. The fact that the final segment of the voice value function exhibited a convex form was not expected. ${ }^{5}$

\section{Test of Hypothesis 3}

Hypothesis 3 predicted that the reference point for subjects from Mexico would occur at a lower voice magnitude level than for subjects from Great Britain, The Netherlands, and the United States. The basis for this prediction were findings by Hofstede (1980) that power distance measures for Great Britain, The Netherlands, and the United States were similar, but quite different than the power distance measure for Mexico. Since previous tests have shown that the shape of the voice value function was not significantly different across each country (i.e., no interaction), examination of the regression coefficients for the indicator variables representing each country provides a test of the third hypothesis. In particular, if the reference point for subjects from Mexico is different than other countries then the regression indicator variables identifying subjects from Great Britain, The Netherlands, and the United States should be negative and statistically significant.

Examination of the regression results reported in the previous section shows that the indicator variables identifying subjects from Great Britain and The Netherlands were negative and statistically significant. However, the indicator variableidentifying subjects from the United States was not statistically significant. These results suggest that, as predicted by Hypothesis 3, the reference points for subjects from Great Britain and The Netherlands were different (i.e., higher) than the reference point for subjects from Mexico. Contrary to predictions of Hypothesis 3, these results also suggest that the reference point for subjects from the United States cannot be declared different than the reference point for subjects from Mexico.

To provide further evidence about these differences, the regression function

${ }^{5}$ Because polynomial regression analyses can besensitiveto outliers several tests for the presence and effect of outliers and influential observations were conducted to determine if the convex portion of the voice function was produced by a few extreme observations. Using both the RSTUDENT and DFBETA measures suggested in Belsley et al. (1980) several influential observations were identified and excluded in several reestimations of the regression function. The revised regression functions were not materially different than the original regression function and the final segment of the voice function remained convex. Hence, it does not appear that outliers or influential observations account for the unexpected shape of the final segment of the voice function. To determine if results varied by country we ran separate regressions for each country and then examined the sign of the second derivative for the final segment of each country's voice function. In all four cases, the final segment of the voice function was found to be convex. Thus, results were similar for subjects from each of the four countries. 
was used to estimate the actual reference point for participants from each country. Recall in prospect theory the reference point is described as theneutral point. Outcomes above the neutral point are coded as gains while outcomes below the neutral point are coded as losses. Assuming that the reference point is a neutral outcome and the value of 5 represents the neutral point on the fairness measure scale used in this study, solving for where the regression function takes on a neutral value of " 5 " yielded the following estimates for each country: for respondents from M exico and the United States the function assumed a value of " 5 " at a voice magnitude level of approximately 3 choices and for respondents from $\mathrm{E}$ ngland and The Netherlands the function assumed a value of " 5 " at a voice magnitude level of approximately 4 or 5 . Hence, the neutral level of voicemagnitudefor subjects from England and The N etherlands $(V=4$ or 5) was somewhat higher than for subjects from Mexico and the United States $(\mathrm{V}=3)$.

To hel $p$ explain this result, subjects' responses to the Hierarchy of Authority scale were analyzed. The purpose of the analysis was to determine whether therewere differences by country in degree of decision centralization (Hierarchy of Authority Scale, Aiken \& Hodge, 1966) that matched results comparing estimated reference points. A one-way ANOVA indicated a significant effect for country, $F(3,121)=3.2, p<.05$. The greatest degree of perceived centralization was in Mexico ( $M=5.8)$, followed by the United States $(M=5.1)$, Great Britain $(M=4.8)$, and The Netherlands $(M=4.6)$. Cell contrasts using Duncan Multiple Range tests indicated significant differences between Mexico versus Great Britain and The Netherlands, $\mathrm{p}<.05$, while the difference between Mexico and the United States was not statistically significant, $p<.11$. These results are consistent with earlier findings that reference points computed from the voice value function of participants from Mexico were lower than participants from England and The Netherlands.

\section{Comparing Participant Expectations with the Voice Value Function} Reference Point

The control condition was included to explore whether some measure of participant expectations could be used to identify the value function reference point. There were two sets of three questions measuring participant expectations. For these questions, a response of 1 indicated an expectation for no voice, 2 an expectation for 5 choices, 3 an expectation for 10 choices, 4 an expectation for 5 choices, and 5 an expectation for 20 choices. As a preliminary step responses to the six questions were analyzed separately in a 2 (order) $\times 2$ (country) design to determine if responses varied by order or country. Respondents from Mexico were not included in this analysis because their questionnaires were administered prior to the development of these questions.

The ANOVA disclosed no significant main effects for order or country; nor was the interaction significant for questions measuring the minimum acceptable, the maximum acceptable, and what participants stated they would settle for. For the question measuring the maximum expected, there was a significant 
order by country interaction, $\mathrm{F}(5,78)=2.1, \mathrm{p}<.05$, and a significant main effect for order, $F(5,78)=9.9, p<01$. For the question describing what would probably happen, there was a main effect due to order, $F(5,78)=8.5, p<.01$. However, analysis indicted no rank reversals on the measured variables that would prevent ignoring order in later analyses.

The lack of a main effect of country or a confounding country $\times$ order interaction allowed data from each country to be combined and overall sample means calculated ( $\mathrm{N}=84$ ). These sample means werethen compared to the previously estimated voice value function reference points to determine which, if any, of the expectation measures best corresponded to the value function reference point. Cell contrasts indicated that the minimum acceptable voice level which approximated 10 choices ( $M=2.9$ ) was significantly different from the minimum expected $(M=3.3)$, what would probably happen $(M=3.3)$, and what participants would settle for $(M=3.3)$, which all exceeded 10 choices. Similarly, theminimum acceptable level of voiceal so differed from the maximum expected $(M=4.4)$ and the maximum acceptable $(M=4.5)$ level of voice that exceeded 15 choices. T values ranged from 2.7 to 13.7 , with $p$ values less than .01 .

Consequently, across the entire sample, the question asking the minimum amount of voice that would be acceptable most closely corresponded to the previously estimated value function reference points. For this expectation measure, respondents indicated that voice that approximated 10 of the 20 choices $(M=2.9, S D=1.0)$ would be minimally acceptable. Given the sample mean, sample standard deviation, and number of respondents a 95\% confidence interval was calculated for this expectation measure. The confidence interval for the population mean ranged from approximately 2.5 to 3.3 on the 5-point scale used. These lower and upper limits correspond approximately to voice magnitude levels of 8 choices and 12 choices, respectively, and do not include the reference points implied by responses of treatment group subjects (voice magnitudes of 3 to 5). Thus, the actual reference points implied from the estimated voice value functions did not match any of the self-reported expectation measures supplied by respondents in the control condition; the reference points were substantively lower than the closest matching expectation measure.

\section{DISCUSSION}

The results of this study are consistent with previous findings reporting a nonlinear value function for voice (Hunton et al., 1998). The nonlinear function was replicated in samples of students from four countries. In addition, the shape of the value function for voice did not differ across the sampled countries. This pattern of results provides support for Hypotheses 1 and 2 in the region of the voice function above the reference point.

As illustrated in Fig. 2, the value functions for respondents in each country were similar in several important ways. First, the largest stepwise increase in fairness occurred when voice increased from mute to voice on 5 of the 20 alternatives. In fact, the increase in fairness that occurred from the mute 
condition to voice on 5 choices was larger than the increase in fairness from voice on 5 choices to voice on all 20 choices. Hence, for our subjects, the majority of the benefit gained from allowing voice occurred at a relatively low voice magnitude. Second, each of the four sets of participants appeared to utilize a reference point in the same general region. As shown in Fig. 2, voice levels were perceived as unfair in the mute condition but changed to neutral or above on the measure of fairness in the region of voice on 5 choices. While perceptions of fairness increased in absolute terms as voice rose from 10 to 15 to 20 of 20 choices, stepwise increases were not statistically significant. Consequently, the beneficial effects of voice did not require full participation of the participants. These results suggest a high degree of correspondence with regard to how fairness is interpreted across different student populations in the four countries studied. This work also confirms findings by Lind et al. (1997), which report an association between participant voice and procedural justice.

An unexpected finding was that the final segment of the voice value function exhibited a convex shape, suggesting increasing marginal value. In general, thepredictions of prospect theory arethat the valuefunction will beconsistently concave in the region above the reference point and this is what Hunton et al. (1998) found. However, Kahneman and Tversky (1979, p. 278) acknowledge that special circumstances may produce convex regions in the portion of the value function that lies above the reference point. In particular they suggest that a value function may exhibit an exceptionally steep rise near a critical value for the commodity of interest. The example used by Kahneman and Tversky is that of an individual who needs $\$ 60,000$ to purchase a house. For this individual, because $\$ 60,000$ is a critical value, the valuefunction for money may exhibit an unusually sharp rise as personal savings approach the value of $\$ 60,000$.

As applied to the current study, our subjects knew from the context of the case scenario that the maximum voice possible was 20 of 20 choices. Because voice on 20 attributes represented voice on $100 \%$ of the available choices, our participants may have perceived voice on 20 choices to be a critical and desirable value. In turn, the prospect of achieving voice on this maximum level appears to have produced a convex region in the gain portion of the value function. This suggests that, in certain circumstances, voice value functions may exhibit convex portions (increasing marginal value) as the magnitude of voice received moves from the penultimate level to the maximum level possible. However, it should be emphasized that these findings are tentative in nature and that more work is needed to confirm this property of the voice value function and to identify the circumstances in which it occurs.

Our data suggest that excess amounts of voice increase fairness perceptions monotonically but in a nonlinear manner. The smaller increases in valuation as voice approached its maximal level are consistent with the asymptoticfunctions describe by Rice et al . (1985) and French et al . (1982). It is also consistent with theorizing by these researchers that excess amounts of a commodity are valued when they areliked (Locke, 1969) or increasesatisfaction in other areas (F rench et al., 1982). In this regard theory and research in procedural justice suggest 
that voice is valued because it may result in more favorable outcomes as well as increases in esteem and inclusion (e.g., Lind et al., 1990). However, the voice functions found in the present study are quite different from the inverted Ushaped function suggested by Rice et al . (1985), French et al . (1982), and Locke (1969). However, one can envision circumstances when the voice value function might exhibit negative marginal returns implicit in the inverted $U$-shaped function. This could occur when increments in voice above a certain point are disliked (Locke, 1969) or when the time devoted to exercising higher levels of voice reduces satisfaction in other areas of an individual's life (F rench et al., 1982).

\section{Generality of the Value Function across Cultures}

As a whole our results indicatea great deal of similarity in the inter pretation of fairness and the value function for voice across difference cultures. The value function for voice was of a similar form and increases in the magnitude of voice were associated with increases in perceived fairness. When self-reported measures of participant expectations were collected, they did not differ among respondents in England, The Netherlands, and the United States. Additionally, these self-reported measures of participant expectations did not match estimates of the reference point estimated from the voice value function.

Differences that did emerge between countries were in the estimation of the actual reference points. Participants from Mexico and the United States appeared to utilize reference points in the range of $\mathrm{V}=3$, while participants from England and The Netherlands utilized reference points in the range of $\mathrm{V}=4$ or 5 , providing partial support for our third hypothesis. Scores on the Hierarchy of Authority (Aiken \& Hodge, 1966) measure were generally consistent with differences in theactual referencepoint. Participants from M exicohad significantly higher centralization of authority scores and a lower estimated reference point than did participants from Great Britain and The Netherlands. Findings of differences in the Hierarchy of Authority scale supports cultural differences among participants sharing elements of a similar student culture, and the consistency of these scores with actual differences in the reference point support the expected cultural differences. However, as noted earlier, caution must be exercised in explaining differences in the reference point based on Hofstede's (1980) power distance dimension. While these findings are consistent with cultural differences in participation reported in other studies (e.g., McFarlin \& Sweeney, 1998) and country differences in power distance identified by Hofstede (1980), this does not mean that power distance was the moderating variable. Countries in our sample differ on a number of other variables including other dimensions of culture identified by Hofstede (1980). Additionally, there seem to be greater similarities than differences in the value function for voice among participants from the four different countries that were examined. Perhaps the use of students as subjects served to minimize potential cultural differences that would be more pronounced in nonstudents. 
Correspondence between the Value Function Reference Point and Seff-

Reported Expectations

This research also investigated whether several alternative measures of participant expectations for voice could account for the actual reference point estimated through regression analysis. To our knowledge, no prior studies have attempted to compare expectations to the actual reference point. Of the six measures of expectations, the measure that most closely corresponded to the reference point was the mi nimal amount of voice that partici pants found acceptable. To this question, participants indicated that voice that approximated 10 of the 20 choices was acceptable ( $\mathrm{M}=2.9$ on the 5 -point scale). This value did not correspond to the reference points estimated from the voice value function ( 3 to 4 choices). Based on these data, there seems to be a discrepancy between what people say is the amount of voice that is needed to be fair and the amount of voice that participants experience as being fair. Perhaps this discrepancy is based on a negotiation strategy. That is, when asked, participants say that they expect more voice in hope that they will get more. Another possibility is that when individuals are asked to identify a "fair" outcome, their thought processes focus on the outcome level that would evoke positive feelings. In the context of a value function, a positive outcome that is in the gain domain, above the actual reference point, would be considered fair.

Consequently, when asking participants to identify the actual reference point it may be necessary to use a control group to identify a barely fair outcome and a barely unfair outcome, and then use interpolation procedures to infer the actual reference point that falls between these two points. If either of the above explanations are correct, then measurement, rather than theoretical issues, can explain the discrepancy between our measurement of expectations and the theoretical reference point in prospect theory. However, we would like to emphasize the current research may have reveal ed some important nuances when examining participant expectations and the role they play in the psychology of procedural fairness. Perhaps this article can hel p pave the way to exploring this and other interesting issues in the domain of social justice research.

\section{Methodological Considerations}

In the present article the use of scenarios provided the opportunity to use comparable stimulus materials to examine the value function for voice across four countries. However, in the social justice domain, some researchers have been critical of the use of scenarios (e.g., Lind, 1998). They have argued that when onecompares theimpact of voice on attitudes and behavior in experiential studies to the impact that voice has on people reacting to hypothetical scenarios, one finds that people often underestimate the impact of voice when using the latter as opposed to the former research method (see, e.g., Lind \& Tyler, 1988, for a discussion of this underestimation effect). This said, however, it should be pointed out that there is also a considerable body of research evidence that shows convergent findings when using different research methods (for an overview, see Lind \& Tyler, 1988). Studies conducted by Van den Bos, Lind, 
and their colleagues, for example, typically show similar results of voice when using scenario and experiential experiments (see, e.g., Van den Bos, Lind, Vermunt, \& Wilke, 1997; Van den Bos, Wilke, \& Lind, 1998; Van den Bos, Wilke, Lind, \& Vermunt, 1998).

Of course, previous convergence of scenario and experiential studies does not prove the accuracy of a methodology. This is important, in part, because there are also studies in which sharp differences between methods were observed. Most striking in this respect is the LaTour (1978) study. Interested in conflict resolution using inquisitorial and adversarial procedures, LaTour examined diifferences between single investigator inquisitorial, double investigator inquisitorial, assigned adversary, and choice adversary procedures. Especially relevant for this study, LaTour predicted a linear effect, in which participants' reactions-thelitigants in the dispute-would betheleast positivein the single investigator inquisitorial and the most positive in the choice adversary condition. With respect to observers' reactions-participants witnessing the trial-LaTour predicted a nonlinear effect. He argued that observers, like participants, might be concerned that a single investigator could be biased and/or would be unable to fully appreciate each side of the case. Separation of presentations (single versus double investigator condition) should eliminate these concerns, increasing observers' satisfaction and perceptions of fairness. Addition of outcome correspondence between client and legal representative (assigned adversary condition) and the addition of choice (choice adversary condition) were not expected to increase the favorability of observer evaluations, however. This hypothesis was derived from Heider's (1958) and J ones and Nisbett's (1971) discussions that observers tend to focus on the behaviors of actors ignoring background factors related to that behavior. "While separation of presentations involves a difference in the behavior of attorneys, outcome correspondence and choice are background factors. As a result, these latter factors are not expected to be particularly salient features of a procedure for observers and are not expected to impact on observer satisfaction and fairness judgments" (LaTour, 1978, pp. 1533-1534). Consequently LaTour predicted observers' reactions would be less positive in the single investigator condition than in the other three procedure conditions.

Generally speaking, findings were supportive for LaTour's hypotheses and reveal ed linear effects among partici pants and nonl inear effects among observers. These findings are important in that they suggest the possibility that the current study might have uncovered a linear voice value function if experiential methods were used. It should be noted, however, that there are some important differences between the LaTour study and the study we presented here. First, subjects in LaTour's study experienced treatment effects experientially as either courtroom defendants or observers, while subjects in our scenario study were told to assume the events were happening to them. Second, LaTour's manipulations involved variations al ong multiple dimensions rather than variations in magnitude along a single dimension as in our study. Third, LaTour predicted different effects for participants and observers due to the different roles assumed by these subjects; whereas in our study the predicted nonlinear 
effects were based on the expectation of a declining marginal effect as the magnitude of voice increased. Given these key differences in methodology, concerns that scenario-based findings underestimate the impact of voice-based participation will remain open until future research can be conducted to resolve the complex issues involved.

It should be noted here that while it is possible that the methodology used in the current study may have affected our findings, what matters most is that the present study suggests that particular effects may occur. Although we predicted these effects on the basis of a well-established theory, other research methods will be needed to show that the effects reported in this paper occur in real-world situations (e.g., by means of field experiments) and to provide an indication of the frequency with which these effects occur in everyday life (e.g., by means of surveys). However, we hope to have shown in this article that our findings may have implications for procedural justice research that are quite surprising and that may stimulate future research avenues. As research accumulates concerning the psychology of voice, as it has in this study and in other studies (see, e.g., Lind et al., 1990; Tyler, 1987), we begin to understand the conditions when the effects of voice are not very strong, why they occur at all, and why they are so potent when they do occur. This knowl edgein turn promises to advance our understanding of fundamental issues in the social psychology of justice and of the role of justice-related phenomena in basic social relations.

\section{Conclusions}

This study demonstrates the usefulness of treating voice as a commodity. While treating voice as a commodity does not consider symbolic aspects of voice, as with other commodities, the marginal increment in value generally diminishes as the magnitude of voice increases. In a cost benefit analysis, participation levels below full participation may not be warranted. Consequently, this approach offers a more a precise way to understand the value associated with different levels of voice-based participation. While this study examined voice primarily in the region above the reference point (domain of gains), future research might focus on the region below the reference point (domain of losses) for a similar S-shaped function. Additionally, this research suggests that viewing voice as a commodity is useful in examining and understanding the cultural differences and similarities in the value attached to voice.

\section{REFERENCES}

Aiken, M., \& Hodge, J . (1966). Organizational alienation: A comparative analysis. American Sociological Review, 31, 479-507.

Baldwin, T. T., Magjuka, R. J ., \& Loher, B. T. (1991). The perils of participation: Effects of choice of training on trainee motivation and learning. Personnel Psychology, 44, 51-65.

Belsley, D., Kuh, E., \& Welsch, R. (1980). Regression diagnostics: Identifying influential data and sources of collinearity. New York: Wiley.

Brockner, J ., \& Greenberg, J . (1990). The impact of layoffs on survivors: An organizational justice perspective. In S. J . Carroll (Ed.), Applied social psychol ogy and organizational settings. Hillsdale, NJ : Earbaum. 
Brockner, J ., \& Wiesenfeld, B. M. (1996). An integrative framework for explaining reactions to decisions: Interactive effects of outcomes and procedures. Psychol ogical Bulletin, 10 (2), 189-208.

Coalter, T., \& Price, K. (1997). When voice matters: The influence of organizational traditions, sources of the tradition and historical outcomes on perceived fairness. Unpublished paper.

Coren, S., Ward, L., \& Ellis, J . (1994). Sensation and perception (4th ed.). New York: Harcourt Brace College.

Cook, J . D., Hepworth, S. J ., Wall, T. D., \& Warr, T. D. (1981). The work: A compendium and review of 249 measures and their uses, London: Harcourt Brace J ovanovich.

Cooper, M. R., \& Wood, M. T. (1974). Effects of member participation and commitment in group decision making on influence, satisfaction and decision riskiness. J ournal of Applied Psychology, 59, 127-134.

Doll, W. J ., \& Torkzadeh, G. (1988). The measurement of end-user computing satisfaction. MIS Quarterly, 12 (February), 258-274.

Earley, P. C. (1999). Playing follow the leader: Status-determining traits in relation to collective efficacy across cultures. Organizational Behavior and Human Decision Processes, 80, 192-212.

Earley, P. C., \& Lind, E. A. (1987). Procedural justice and participation in task selection: The role of control in mediating justice judgments. J ournal of Personality and Social Psychology, 52, 1148- 1160.

Eylon, D., \& Au, K. Y. (1999). Exploring empowerment cross-cultural differences along the power distance dimension. International J ournal of Interculture Relations, 23, 373- 385.

Folger, R. (1977). Distributive and procedural justice: Combined impact of "voice" and improvement on experienced inequity. J ournal of Personality and Social Psychology, 35, 108-119.

Folger, R. (1986). Rethinking equity theory: A referent cognition model. In H. W. Bierhoff, R. L. Cohen, \& J . Greenberg (Eds.), J ustice in social relations (pp. 145-162). New York: Plenum.

Folger, R. (1987). Reformulating the reconditions of resentment: A referent cognitions model. In J . C. Masters \& W. P. Smith (Eds.), Social comparison, justice, and relativedeprivation: Theoretical, empirical, and policy perspectives (pp. 183-215). Hillsdale, NJ : Erlbaum.

Folger, R., \& Cropanzano, R. (1998). Organizational justice and human resource management. Thousand Oaks, CA: Sage.

Folger, R., Rosenfield, D., \& Robinson, T. (1983). Relative justification and procedural justification. J ournal of Personality and Social Psychology, 45, 268-273.

French, R. P., J r., Caplan, R. D., \& Harrison, R. V. (1982). Themechanisms of job stress and strain. New York: Wiley.

Galanter, E. (1962). The direct measurement of utility and subjective probability. American J ournal of Psychology, 75, 208-220.

Greenberg, J . (1990). Organizational justice: Yesterday, today and tomorrow. J ournal of Management, 16, 399-432.

Greenberg, J ., \& Folger, R. (1983). Procedural justice and the fair process effect in groups and organizations. In P. B. Paulus (Ed.), Basic group processes (pp. 235-256). New York: Springer-Verlag.

Greenhaus, J . H., Seidel, C., \& Marinis, M. (1983). The impact of expectations and values on job attitudes. Organizational Behavior and Human Performance, 31, 394- 417.

Heider, F. (1958). The psychology of interpersonal relations. New York: Wiley.

Hofstede, G. (1984). The cultural relativity of the quality of life concept. The Academy of Management Review, 9, 389-398.

Hofstede, G. (1980). Motivation, leadership, and organization: Do American theories apply abroad? Organizational Dynamics (Summer), 42-63.

Hunton, J . E., \& Price, K. H. (1997). Effects of the user participation process and task meaningfulness on key information system outcomes, Management Science, 43(6), 797-812.

Hunton, J. E., Price, K. H., \& Hall, T. W. (1996). A field experiment examining the effects of 
membership in voting majority and minority subgroups and the ameliorating effects $f$ postdecisional voice. J ournal of Applied Psychology, 81, 806-812.

Hunton, J . E., Hall, T. W., \& Price, K. H. (1998). The value of voice in participative decision making. J ournal of Applied Psychology, 83(5), 788-797.

Kahneman, D. (1992). Reference points, anchors, norms, and mixed feelings. Organizational Behavior and Human Decision Processes, 52(2), 296-312.

Kahneman, D., \& Tversky, A. (1979). Prospect theory: An analysis of decisions under risk. Econometrica, 47, 263-291.

Kahneman, D., \& Tversky, A. (1982). The simulation heuristic. In D. Kahneman, P. Slovic, \& A. Tversky (Eds.), J udgement under uncertainty: Heuristics and biases New York: Cambridge Univ. Press.

Karambayya, R., \& Brett, J . M. (1989). Managers handling disputes: Third-party roles and perceptions of fairness. Academy of Management J ournal, 32, 687-704.

Kras, E. S. (1995). Management in two cultures: Bridging the gap between U.S. and Mexican managers (rev. ed.). Yarmouth, ME: Intercultural. Latane, B. (1981). The psychology of social impact. American Psychologist, 36, 343- 356.

LaTour, S. (1978). Determinants of participant and observer satisfaction with adversary and inquisitorial modes of adjudication. J ournal of Personality and Social Psychology, 36(12), 1531- 1545.

Leung, K., \& Lind, E. A. (1986). Procedural justice and culture: Effects of culture, gender, and investigator status on procedural preferences. J ournal of Personality and Social Psychology, 50(6), 1134-1140.

Lind, E. A. (1995). J ustice and authority relations in organizations. In R. S. Cropanzano \& K. M. Kacmar (Eds.), Organizational politics, justice and support: Managing the social climate of the workplace (pp. 83-97). Westport, CT: Quorum Books.

Lind, E. A. (1998, May). Methods in procedural justice. Paper presented at theSeventh International Conference on Social J ustice Research, Denver, CO.

Lind, E., Kanfer, R., \& Earley, P. C. (1990). Voice, control and procedural justice: Instrumental and noninstrumental concerns in fairness judgments. J ournal of Personality and Social Psychology, 59, 952-959.

Lind, E. A., \& Tyler, T. R. (1988). The social psychology of procedural justice. New York: Plenum.

Lind, E. A., Tyler, T. R., \& Huo, T. J . (1997). Procedural context and culture: Variation in the antecedents of procedural justice judgements. J ournal of Personality and Social Psychology, 73, 767-780.

Locke, E. A. (1969). What is job satisfaction? Organizational Behavior and Human Performance, 4, 309- 336.

Maier, N. R. (1973). Psychology in industrial organizations. Boston: Houghton Mifflin.

Miceli, M. P., \& Lane, M. C. (1991). Antecedents of pay satisfaction: A review and extension. In K. Rowland \& G. R. Ferris (Eds.), Research in personnel and human resources management (pp. 235-309). Greenwich, CT: J Al Press.

McEvoy, G. M., \& Cascio, W. R. (1990). The U.S. and Taiwan: Two different cultures look at performance appraisal. In K. M. Rowland \& G. R. Ferris (Eds.), Research in personnel and human resources management (Vol. 6, pp. 201-219). Greenwich, CT: J Al Press.

McFarlin, D. B., Sweeney, P. D., \& Cotton, J . C. (1993). Attitudes toward employee participation in decision-making: A comparison of European and American managers in a U.S. Multinational. Human Resource Management J ournal, 31, 363-383.

McFarlin, D. B., \& Sweeney, P. D. (1998). International management: Trends, challenges, and opportunities. Cincinnati, $\mathrm{OH}$ : South-Western College.

Peterson, R. S. (1999). Can you have too much of a good thing? The limits of voice for improving satisfaction with leaders. Personality and Social Psychology Bulletin, 25(3), 313-324. 
Rice, R. W., McFarlin, D. B., Hunt, R. G., \& Near, J . P. (1985). Organizational work and the perceived quality of life: Toward a conceptual model. Academy of Management J ournal, 10, 296-310.

Shane, S., Venkataraman, S., \& MacMillan, I. (1995). Cultural differences in innovation championing strategies. J ournal of Management, 21, 931-952.

Smith, P. B., Dugan, S., Peterson, M. F., \& Leung, K. (1998). Individualism: Collectivism and the handling of disagreement: A 23 country study. International J ournal of Intercultural Relations, 22, 351-367.

Sondergaard, M. (1994). Hofstede's consequences: A study of reviews, citations and replications. Organization Studies, 15, 447-456.

Stohl, C. (1993). European managers' interpretations of participation: A semanticnetwork analysis. Human Communication Research, 20, 97-117.

Stumpf, S. A., Zand, D. E., \& Freedman, R. D. (1979). Designing groups for judgmental decisions. Academy of Management Review, 4, 589-600.

Thibaut, J ., \& Walker, L. (1975). Procedural justice: A psychological analysis. Hillsdale: Erlbaum. Tyler, T. R. (1987). Conditions leading to value-expressive effects in judgments of procedural justice: A test of four models. J ournal of Personality and Social Psychology, 52, 333-344.

Tyler, T. R., \& Lind, E. A. (1992). A relational model of authority in groups. Advances in Experimental Social Psychology, 25, 115-191.

Van den Bos, K., Lind, E. A., Vermunt, R., \& Wilke, H. A. M. (1997). How do I judge my outcome when I do not know the outcome of others?: The psychology of the fair process effect. J ournal of Personality and Social Psychology, 72, 1034- 1046.

Van den Bos, K., Vermunt, R., \& Wilke, H. A. M. (1996). The consistency rule and the voice effect: The influence of expectations on procedural fairness judgments and performance. European J ournal of Social Psychology, 26, 411-428.

Van den Bos, K., Wilke, H. A. M., \& Lind, E. A. (1998). When do we need procedural fairness? The role of trust in authority. J ournal of Personality and Social Psychology, 75, 1449- 1458.

Van den Bos, K., Wilke, H. A. M., Lind, E. A., \& Vermunt, R. (1998). Evaluating outcomes by means of the fair process effect: Evidence for different processes in fairness and satisfaction judgments. J ournal of Personality and Social Psychology, 74, 1493- 1503.

Vroom, V. H., \& J ago, A. R. (1988). The new leadership. Englewood Cliffs, NJ : Prentice-Hall.

Received May 24, 1999; published online November 7, 2000 